Faculdade de Ciências Econômicas UFRGS
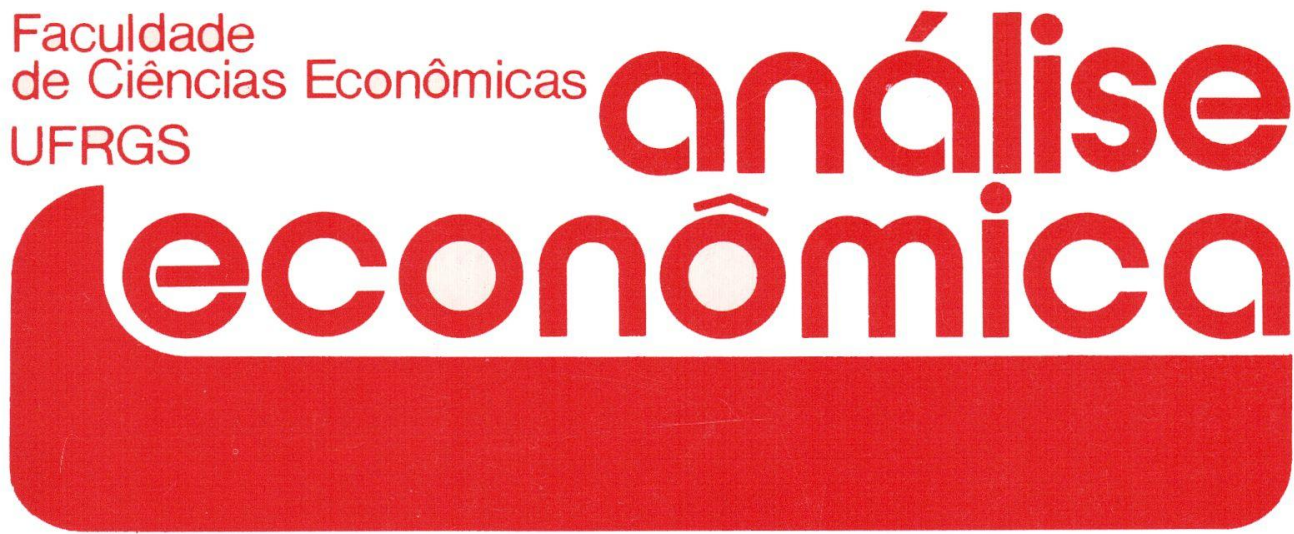

- INDEXAÇĀo SALARIAL: UMA ABORDAGEM MACROECONÓMICA Jo Anna Grav

- AJUSTE NO EMPREGO E PRODUTIVIDADE NA DECADA DE OITENTA

Carlos Antônio Luque José Paulo Zeeteno Chahad

- O CONSUMIDOR KEYNESIANO Marcelo Córtes Neri

- HETEROGENEIDADE DO TRABALHO E TAXA DE LUCRO EM MARX

Francisco Cribari Neto

- ECONOMIAS DE ESCALA: UMA REVISĀO Jesiel de Marco Gomes

- Concentraçấo bancária no BRASIL

Marcelo Resende

- NOYOS RUMOS PARA O SETOR ELETRICO NO BRASIL

Adriano Pires Rodrigues

Eduardo da Cunha Vianna

- OFERTA E DEMANDA DE FRANGO DE CORTE NO BRASIL

Narciso Gonçalves de Castro et alii

- ANÁLISE ECONÔMICA DA IRRIGAÇÃo DO MILHO

Lúcia M Schirmer

Juvir Luiz Mattuella

- REFLORESTAMENTO NO BRASIL. Carlos José Caetano Bacha

- ESCOLHA DE TECNOLOGIA EM ESTRUTURA DE PRINCIPAL AGENTE Kyle D. Kauffma:

- A QUESTÁo DEMOGRÁfica e A PRAXEOLOGIA

Anton Karl Biedermann et alii
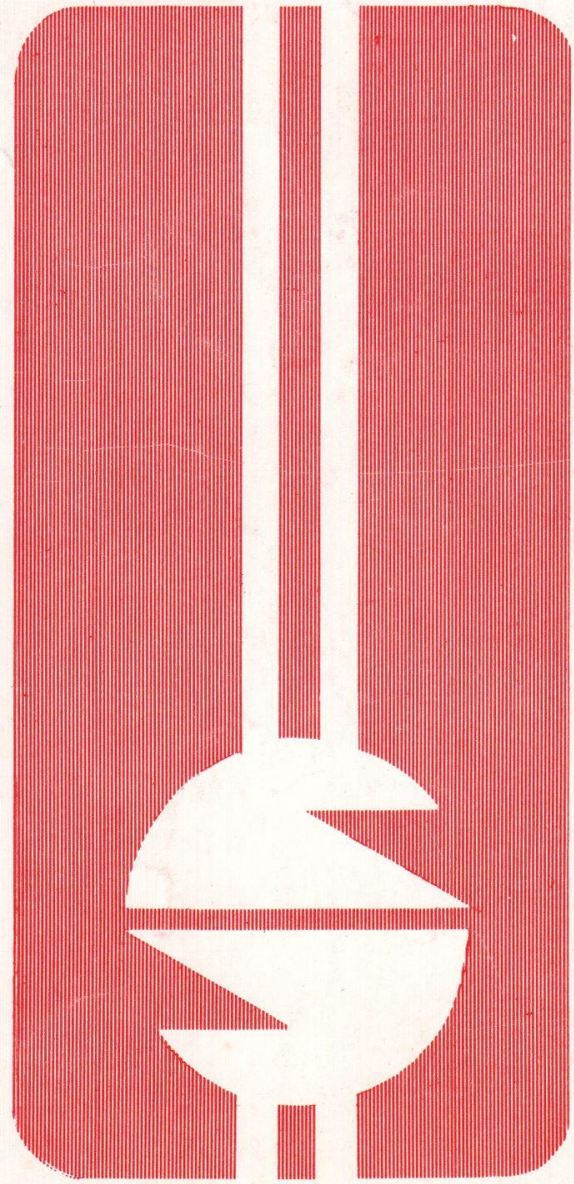
UNIVERSIDADE FEDERAL DO RIO GRANDE DO SUL

Reitor: Prof. Tuiskon Dick

FACULDADE DE CIÉNCIAS ECONÔMICAS

Diretora: Prof ${ }^{\text {a }}$ Yeda Rorato Crusius.

CENTRO DE ESTUDOS E PESQUISAS ECONÔMICAS

Diretor: Reinaldo Ignacio Adams

DEPARTAMENTO DE CIÉNCIAS ECONÔMICAS

Chefe: Prof. Fernando Ferrari Filho

CURSO DE POS-GRADUAÇÄO EM ECONOMIA

Coordenador: Prof. Nali de Jesus de Souza

CURSO DE PÓS-GRADUAÇĀO EM ECONOMIA RURAL

Coordenador: Prof. Atos Freitas Grawunder

CONSELHO EDITORIAL: Achyles Barcelos da Costa, Aray Miguel Feldens, Atos Freitas Grawunder, Carlos Augusto Crusius, Ernani Hickmann, João Rogério Sanson, Juvir Luiz Mattuella, Maria Imilda da Costa e Silva, Nali de Jesus de Souza, Nuno Renan Lopes de Figueiredo Pinto, Otilia Beatriz Kroeff Carrion, Otto Guilherme Konzen, Paulo Alexandre Spohr, Pedro Cezar Dutra Fonseca, Reinaldo Ignacio Adams, Roberto Camps Moraes, Valter José Stülp, Yeda Rorato Crusius, David Garlow (Wharton Econometrics Forecasts Association, E.U.A.), Edgar Augusto Lanzer (UFSC), Eleutério F.S. Prado (USF), Fernando Holanda Barbosa (FGV/RJ), Gustavo Franco (PUC/RJ), Joaquim Pinto de Andrade (UnB), Juan H. Moldau (USP), Werner Baer (Univ. de Illinois, E.U.A.).

COMISSĀO EDITORIAL: Atos Freitas Grawunder, Pedro Cezar Dutra Fonseca, Reinaldo Ignacio Adams e Roberto Camps Moraes.

EDITOR: Nali de Jesus de Souza

SECRETARIA: Maria Ivone de Mello (normalização), Vanete Ricacheski (revisão de textos).

FUNDADOR: Prof. Antônio Carlos Santos Rosa

Os materiais publicados na revista Análise Econômica são de exclusiva responsabilidade dos autores. É permitida a reprodução total ou parcial dos trabalhos, desde que seja citada a fonte.

Aceita-se permuta com revistas congêneres. Aceitam-se, também, livros para divulgação, elaboração de resenhas ou recenșões.

Toda correspondência, material para publicação, assinaturas e - permutas devem ser dirigidos ao seguinte destinatário:

\section{PROF. NALI DE JESUS DE SOUZA}

Revista Análise Econômica

Av. João Pessoa, 52

CEP 90040-000 - PORTO ALEGRE (RS), BRASIL

Telefones: (051) 228-1633 - 224-6024 ramais 3440 e 3348

Fax: (051) 225-1067 


\title{
MENSURAÇÃO DA CONCENTRAÇÃO BANCÁRIA NO BRASIL, 1970/86*
}

\author{
Marcelo Resende
}

\section{SINOPSE}

Ește artigo pretende quantificar a concentraçăo bancária no Brasil ao longo do perfodo 1970/86 para as variáveis depósitos totais, depósitos a vista, depósitos a prazo, número de agências e emprestimos. Pode-se verificar um grande aumento da concentraç̋̆o no grupo dos bancos comerciais privados, o que reflete, de um lado, medidas governamentais de incentivo e, de outro lado, a natureza cambiante das formas de competiçăo, dado o ambiente inflacionário. Podese esperar que a estabilização do nfvel geral de preços contribufria, ainda que pouco, para o aumento no nivel de concorréncia no setor bancário.

\section{INTRODUÇĀO}

O setor bancário brasileiro tem merecido a atenção de diversos es-

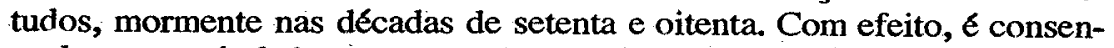
sual que o nivel da concentração banḉria se elevou sunstancialmente, sobretudo na década de setenta. Tal fenômeno certamente reflete, em grande miedida, as polfticas regulatórias de incentivo à concentração desenvolvidas pelo governo. Esse tipo de política remonta à década de quarenta (Ver Melo Filho/1972), mas atingiu seu auge na primeira iietade da década de setenta através da operação da Comissão de Fusões e Incorporaçōes (COFIE), que incentivava fusões e incorporações no setor

* Este trabalho resume o caṕftulo 3 da dissertação de mestrado apresentada a PUC/RJ. O autor gostaria de agradecer o auxflio do estagiário Raul Zenha e o suporte financeiro do PNPE' IPEA e da Fundaçāo Guilherme Guinle.

** Professor assistente do Departamento de Economia da FEA/UFRJ.

\begin{tabular}{|l|l|l|l|l|}
\hline ANÁLISE ECONŌMICA : & ANO 10 & No 17 & MARÇO/92 & P.89-107 \\
\hline
\end{tabular}


bancário, mediante a concessão de vantagens tributárias. ${ }^{1} \mathrm{O}$ principal argumento subjacente a tais políticas, de base empírica duvidosa, era o de que os bancos remanescentes de maior porte realizariam economias de escala, o que acabaria sendo repassado em termos de patamares de taxas de juros mais reduzidos. De qualquer forma, à parte da discussão das causas da concentração bancária, foram empreendidos esforços no sentido de quantificar essa concentração nos trabalhos de Bouzan (1972), Meirelles (1975), Fonseca \& Sanvicente (1977), Castro (1981), Tavares (1981), Corrêa (1985) e Marques (1985). ${ }^{2}$ Tais estudos, via de regra, apontam para um aumento significativo da concentração, ao menos na primeira metade da década de setenta. Contudo, pecam em geral pela utilização de medidas de concentração toscas, pela consideração de perfodos espaçados e pelo nível de agregação dos dados. Nesse sentido, o objetivo do presente trabalho é o de preencher uma lacuna na literatura, através de um esforço de quantificação mais rigoroso.

A dificuldade em se definir o produto de um banco, serve de motivação para uma análise desagregada em termos das variáveis depósitos a vista, depósitos a prazo, emprestimos e número de agências. $O$ trabalho está organizado da seguinte forma. A segunda seção apresenta uma breve digiessão acerca do fndice de concentração a ser utilizado e suas decomposiçōes. A terceira seção apresenta os resultados da mensuração da concentração bancária para os bancos comerciais privados. A quarta e última seção apresenta os comentários finais.

\section{O INDICE DE HERFINDAHL E SUAS DECOMPOSIÇÖES}

Grande parte dos estudos anteriormente mencionados fez uso das chamadas razōes de contração que, dentre outros problemas, desconsidera mudanças nas posiçōes relativas das firmas não inclurdas no indice. Tendo em vista dificuldades dessa ordem, far-se-á uso do índice de Herfindahl definido como:

$$
H=\sum_{i=1}^{n} P_{i}^{2}
$$

onde $p_{1}$ representa a parcela da i-ésima firma no total da variável sob consideração. É fácil mostrar que os limites de tal f́ndice situam-se entre $1 / n$ e 1 , no caso em que as participaçöes de mercado estão igualmente distriburdas e quando só existe uma firma, respectivamente. Esse indice pode ser ajustado de modo a tornar a amplitude de variação constante, para tanto basta dividir $\mathrm{H}$ pela amplitude possfvel de varia-

1. Para maiores detalhes ver Marques (1985) e Tavares \& Carvalheiro (1985).

2 Para uma resenha desses estudos, ver Resende (1989), capítulo 2. 
ção (n-1)/n), o que nos dá:

$$
H^{\prime}=\frac{n}{n-1} \quad H
$$

Essa variante do fndice tem amplitude de variação igual a 1, mas seus limites inferior e superior dependem do número de firmas. Srivastava \& Aggarwal (1979) sugerem outra versão ajustada do índice de Herfindahl, cujos limites independem do número de firmas e, assim, seria mas conveniente para comparações intertemporais:

$$
H^{\prime \prime}=\frac{1}{n-5}[\mathrm{nH}-1] \quad \text { para } \mathrm{n}>1
$$

Os valores extremos de $\mathrm{H}^{\prime \prime}$, obtidos a partir da substituição dos limites anteriormente mencionados na fórmula acima, permite concluir que esse se situa entre 0 e $1 .^{3}$

Outra possibilidade, de grande apelo intuitivo, consiste na consideração do chamado número equivalente de firmas(ne) definido como o número de firmas de igual tamanho compatível com o valor observado do Índice. É fácil mostrar que esse indicador $€$ igual ao inverso do f́ndice de Herfindahl.

Ádicionalmente, o Indice de Herfindahl, em sua formulação original, permite uma conveniente decomposição. ${ }^{4}$ Ilustramos tal possibilidade pela decomposição da concentração nos depósitos totais em termos da concentração nos depósitos a vista e a prazo.

$$
H D_{t}=\frac{1}{\left(D_{t}\right)^{2}} \sum_{i=1}^{n} D_{i t} D V_{i t}+\frac{1}{\left(D_{t}\right)^{2}} \sum_{i=1}^{n} D_{i t} D P_{i t}
$$

onde:

$\mathrm{DV}_{\text {it }}$ é volume de depósitos a vista do banco i no perfodo $t$

DP it idem para depósitos a prazo

$D_{\text {it }}$ idem para depósitos totais

$$
D_{t}=\sum_{i=1}^{n} D_{i t}
$$

Pode-se interpretar a primeira e segunda parcelas, respectivamente, como a contribuição dos depósitos a vista e dos depósitos a prazo para a

3 Para uma discussäo detalhada sobre o indice de Herfindahl, ver Resende (1990).

4 Ver Srivastava \& Aggarwal (1979), op. cit. 
concentração nos depósitos totais.

Outro ponto de interesse, refere-se à decomposição dos índices de concentração segundo as contribuiçóes dos bancos oficiais e privados. Nesse caso, a decomposiçāo é direta, cabendo apenas identificar a participação desses grupos de bancos no total do fndice.

Nas seções seguintes, passamos a apresentar os resultados por meio de gráficos; as tabelas com o conjunto completo de medidas de concentração aparecem no apêndice.

\section{MENSURAÇĀo da CONCENTRAÇĀo}

\subsection{Introdução}

Os índices de concentração para as variáveis de estudo foram calculados a partir de dados mensais de balancetes individuais de bancos disponfveis na Revista Bancária Brasileira, com os quais foram geradas medias trimestrais que serviram de base para o cálculo dos f́ndices. Os dados, referindo-se especificamente ao número de agências, foram obtidos júnto ao Departamento de Cadastro do Banco Central em bases semestrais para o perfodo 1980/87.

Podemos enumerar algumas dificuldades associadas a estudos anteriores que motivaram um esforço de quantificação da concentração mais cuidadoso: i) Uso de medidas toscas; nesse particular Meirelles (1975), Castro (1981) e Corrêa (1985) são exceções;

ii) Cálculo de f́ndices para perfodos espaçados. Nesse tocante, Bouzan (1972), Tavares (1983) estão isentos de crítica, todavia recaem na critica apresentada em (i);

iii) Utilização de dados referentes a saldo de fim de ano:

iv) Consideraç̃̃o exclusiva da totalidade dos bancos, como fizeram Fonseca \& Sanvicente (1977).

A crftica expressa em (ii) É particularmente relevante se existe a pretensão de discutir econometricamente os determinantes da concentração Bancária. ${ }^{5} \mathrm{~A}$ crítica (iii) pode ser inócua se não se espera que as eventuais distorções contábeis afetem bancos distintos de forma particularmente diferenciada. Por outro lado, a crftica (iv) revela-se fundamental, tendo em vista a hipStese de que os fatores que norteiam as decisões dos segmentos públicos e privados seriam essencialmente distintos. Adicionalmente, as medidas regulatorias do governo visavam essencialmente estimular de forma indireta a eficiência do setor privado (com vistas à redução da taxa de juros). De fato, os bancos públicos por estarem sob

5 Ver Resende (1989), capítulo 4, para um esforço dessa natureza. 
controle de esferas governamenais não exigiriam medidas indiretas de incentivo à eficiência. Tendo em mente tais consideraçōes, justifica-se que concentremos nossa atenção no segmento dos bancos comerciais privados, dada a distorção que seria implicada na análise, sobretudo pela presença do Banco do Brasil. Para se ter uma idéia, o Banco do Brasil chegou a responder por $45 \%$ do total de empréstimos, cabendo salientar que ele atua maciçamente ná concessão de crédito subsidiado à agricultura. Convem, contudo, sumariar as principais conclusões referenes a totalidade dos bancos conforme estudado por Resende (1989):

a) as flutuações dos indices de concentração referentes aos depósitos a vista e empréstimos refletem, via de regra, as flutuações nas participações dos bancos públicos;

b) não se observa o propalado aumento da concentração. Com efeito, a concentração nos depóstios a vista chega a cair à metade do nível máximo observado no inf́cio da década de setenta;

c) observa-se uma contínua redução da participação dos depósitos a vista na explicação da concentração nos depósitos totais.

De t३ido modo, face às consideraçōes anteriormente feitas, essas conclusões revelam-se de interesse limitado, fazendo-se necessário que nos detenhamos no grupo dos bancos comerciais privados a partir da próxima seção.

\subsection{Mensuração da Concentração-Bancos Comerciais Privados}

A observação da evolução da concentração restrita ao grupo dos bancos comerciais privados permite-nos afirmar que aquela se elevuu substancialmente. Os Índices de concentração para depósitos tntais, depósitos a vista, depósitos a prazo e as decomposições relevantes aparecem na tabela 1. O gráfico 1 mosíua a evolução da concentração nos depбsitos totais e pode-se depreender do mesmo um significarivo incremento da concentração. De fato, já na primeira metade da década de setenta, o nfvel de concentração mais do que dobra. 


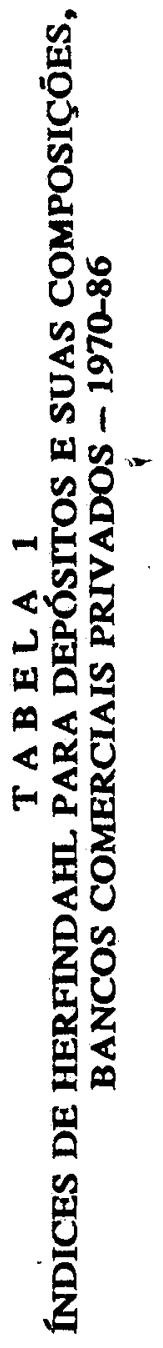

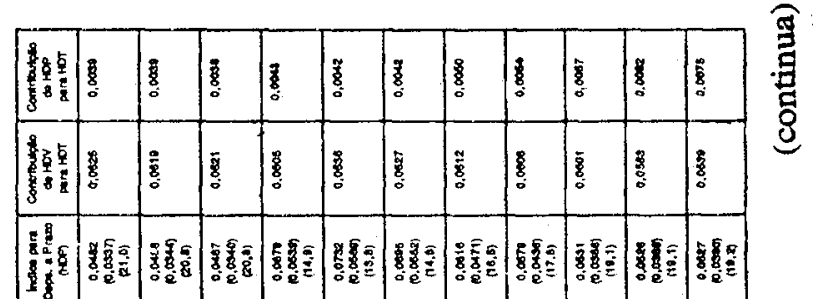

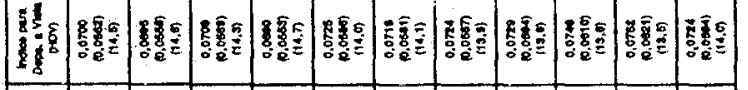

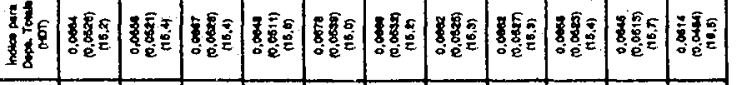

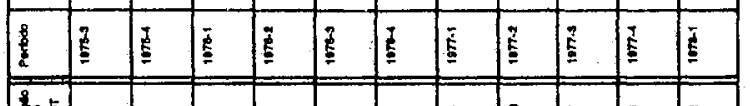

\begin{tabular}{|c|c|c|c|c|c|c|c|c|}
\hline 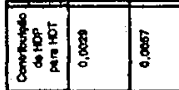 & $\frac{1}{3}$ & \begin{tabular}{l|l}
5 \\
5
\end{tabular} & $1^{8}$ & 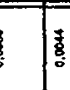 & & $\frac{18}{2}$ & & \\
\hline $\mid \begin{array}{ll}5 \\
\end{array}$ & $\frac{1}{8}$ & \begin{tabular}{|l|l}
5 \\
5
\end{tabular} & 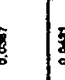 & 1 & & & & \\
\hline
\end{tabular}

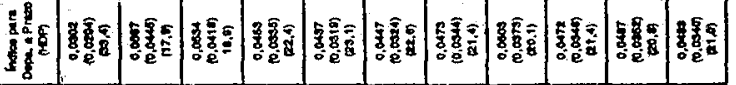

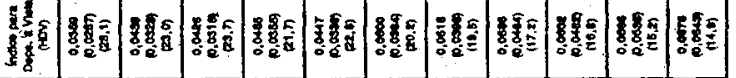

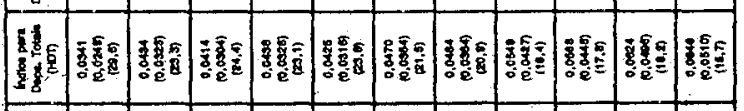

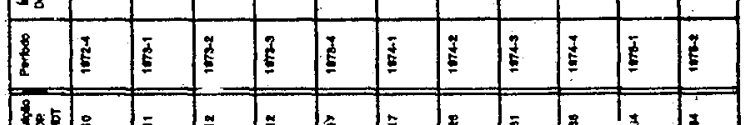

\begin{tabular}{|c|c|c|c|c|c|c|}
\hline 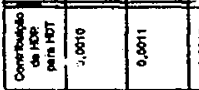 & \begin{tabular}{|l|l|}
5 & 5 \\
5 & 5 \\
\end{tabular} & \begin{tabular}{l|l}
\multirow{2}{*}{$\bar{z}$} \\
\end{tabular} & 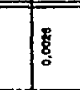 & $\frac{5}{s}$ & & \\
\hline 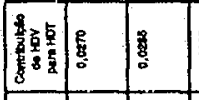 & \begin{tabular}{|l|l|}
5 & E \\
\end{tabular} & \begin{tabular}{|l|l}
5 & 1 \\
\end{tabular} & s & & & 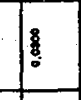 \\
\hline & & & 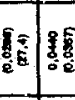 & & & 烈 \\
\hline & & & 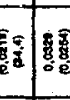 & & & 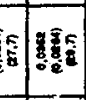 \\
\hline 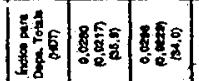 & & & & & & 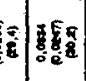 \\
\hline $\mid$\begin{tabular}{|l|l|}
$\underline{z}$ \\
\end{tabular} & & & & & & \\
\hline
\end{tabular}




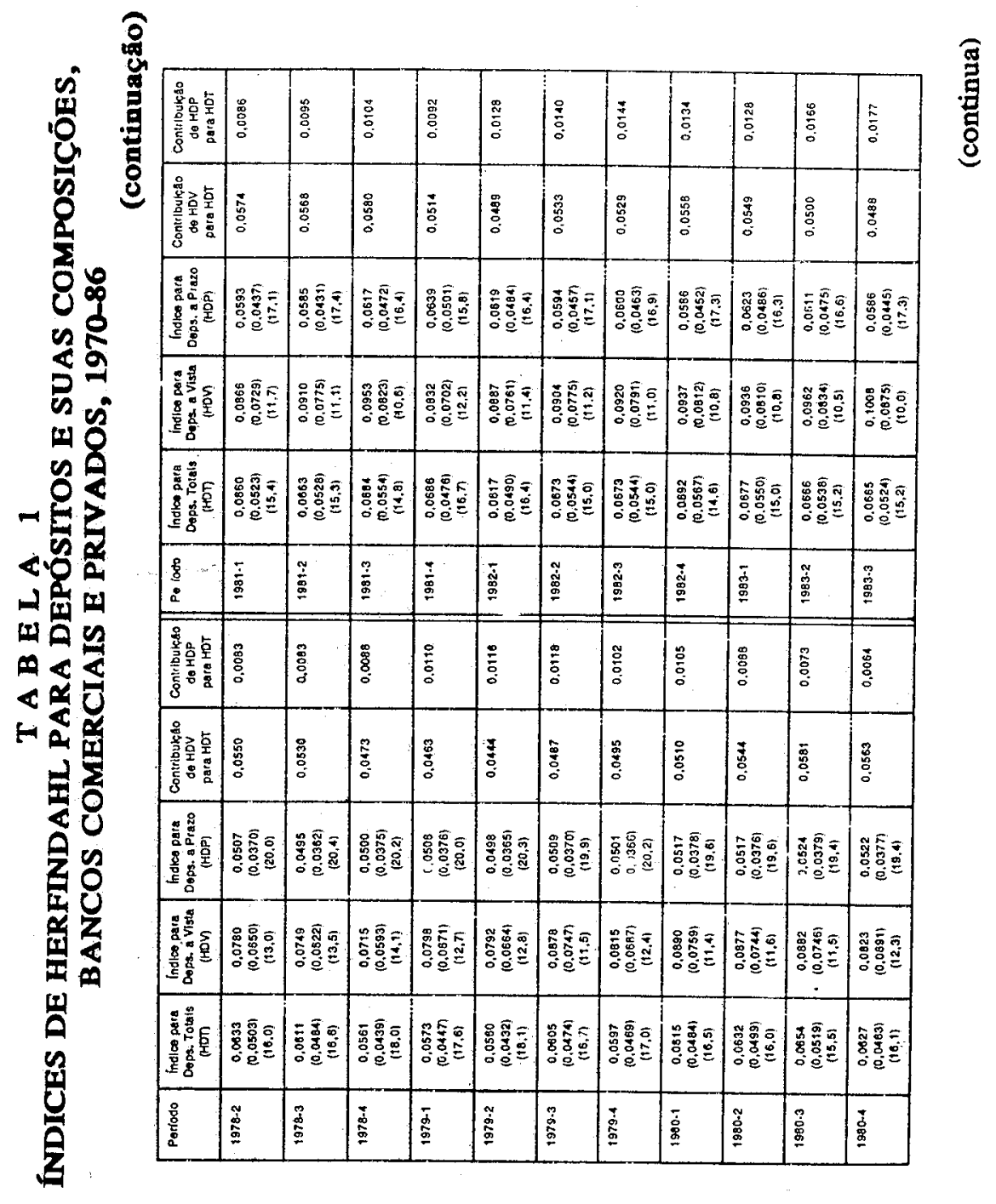




\section{T A B E L A \\ INUICES DE HERFINDAHL PARA DEPÓSTTOS E SUAS COMPOSIÇŌES, BANCOS COMERCIAIS E PRIVADOS, 1970-86 (conclusāo)}

\begin{tabular}{|c|c|c|c|c|c|c|}
\hline & Perlodo & $\begin{array}{l}\text { Indice para } \\
\text { Deps. Totais } \\
\text { (HDT) }\end{array}$ & $\begin{array}{c}\text { Indice para } \\
\text { Deps. a Vista } \\
\text { (HDV) }\end{array}$ & $\begin{array}{c}\text { indice para } \\
\text { Deps. a Prazo } \\
\text { (HDP) }\end{array}$ & $\begin{array}{c}\text { Contribuiçáo } \\
\text { de HDV } \\
\text { para HDT }\end{array}$ & $\begin{array}{l}\text { Contríbulçá } \\
\text { de HDP } \\
\text { para HDT }\end{array}$ \\
\hline & 1983.4 & $\begin{array}{c}0.0635 \\
(0.0500) \\
(16,0)\end{array}$ & $\begin{array}{c}0.1024 \\
(0,0888) \\
(9,9)\end{array}$ & $\begin{array}{c}0.0525 \\
(0.0382) \\
(19,3)\end{array}$ & 0.0461 & 0.0174 \\
\hline & 1984.1 & $\begin{array}{c}0.0585 \\
(0.0442) \\
(17,3)\end{array}$ & $\begin{array}{c}0.1100 \\
(0.0957) \\
(9,2)\end{array}$ & $\begin{array}{c}0.0560 \\
(0.0413) \\
(18,1)\end{array}$ & 0.0357 & 0.0228 \\
\hline & $1984-2$ & $\begin{array}{c}0,0550 \\
(0,0419) \\
(18,4)\end{array}$ & $\begin{array}{c}0.1071 \\
(0.0940) \\
(9,4)\end{array}$ & $\begin{array}{c}0,0480 \\
(0,0343) \\
(21.1)\end{array}$ & 0.0318 & 0.0232 \\
\hline & $1984-3$ & $\begin{array}{c}0.0635 \\
(0.0492) \\
-\quad(16.0)\end{array}$ & $\begin{array}{c}0.1055 \\
(0.0912) \\
(9.6)\end{array}$ & $\begin{array}{c}0.0529 \\
(0.0380) \\
(19,2)\end{array}$ & 0,0323 & 0,0312 \\
\hline & 1984.4 & $\begin{array}{c}0.0602 \\
(0.0469) \\
(16.8)\end{array}$ & $\begin{array}{c}0.1058 \\
(0.0925) \\
(9.6)\end{array}$ & $\begin{array}{c}0.0494 \\
(0.0350) \\
(20.5)\end{array}$ & 0,0304 & 0,0298 \\
\hline & $1985-1$ & $\begin{array}{c}0,0590 \\
(0,0447) \\
(17,2)\end{array}$ & $\begin{array}{c}0,1126 \\
(0,0983) \\
(9,0)\end{array}$ & $\begin{array}{c}0.0498 \\
(0,0350) \\
(20,4)\end{array}$ & 0,0256 & 0.0334 \\
\hline & $1985-2$ & $\begin{array}{c}0.0647 \\
(0,0510) \\
(15,6)\end{array}$ & $\begin{array}{c}0.1180 \\
(0.1043) \\
(8,6)\end{array}$ & $\begin{array}{c}0.0522 \\
(0,0378) \\
(19,4)\end{array}$ & 0.0279 & 0.0368 \\
\hline & $1985-3$ & $\begin{array}{c}0.0697 \\
(0.0560) \\
(14,5)\end{array}$ & $\begin{array}{c}0.1199 \\
(0,1062) \\
(0.4\rangle\end{array}$ & $\begin{array}{c}0.0562 \\
(0.0419) \\
(18,0)\end{array}$ & 0.0309 & 0.0388 \\
\hline & 1985.4 & $\begin{array}{c}0.0793 \\
(0,0646) \\
(12,4)\end{array}$ & $\begin{array}{c}0.1320 \\
0.1173 \\
(7.7)\end{array}$ & $\begin{array}{l}0.0610 \\
0.0456 \\
(16.6)\end{array}$ & 0.0398 & 0.0395 \\
\hline & 19861 & $\begin{array}{c}0.0716 \\
(0.0571) \\
(14,2)\end{array}$ & $\begin{array}{c}0.1185 \\
(0.1040) \\
(8,6)\end{array}$ & $\begin{array}{c}0.0581 \\
(0.0428) \\
(175)\end{array}$ & 0.0339 & 0.0377 \\
\hline & 1986.2 & $\begin{array}{c}0.0894 \\
(0.0744) \\
(11.4)\end{array}$ & $\begin{array}{c}0.1500 \\
(0,1351) \\
(6.8)\end{array}$ & $\begin{array}{c}0.0565 \\
(0.0411) \\
(18,0)\end{array}$ & 0.0689 & 0.0205 \\
\hline & $1986-3$ & $\begin{array}{c}0.0850 \\
(0.0702) \\
(11.9)\end{array}$ & $\begin{array}{c}0.1561 \\
(0.1414) \\
(6,5)\end{array}$ & $\begin{array}{c}0.0470 \\
(0.0316) \\
(21.6)\end{array}$ & 0.0647 & 0.0203 \\
\hline & $1986-4$ & $\begin{array}{c}0.0724 \\
(0.0577) \\
(14.0)\end{array}$ & $\begin{array}{c}0.1489 \\
(0.1342) \\
(6,8)\end{array}$ & $\begin{array}{c}0.0450 \\
(0,0296) \\
(22,6)\end{array}$ & 0.0499 & 0.0225 \\
\hline
\end{tabular}

NOTA: Os valores contidos em cada célula são respectivamente: o Indice de Herfindahl ajustado relativamente à amplitude de variaçāo ( $\left.\mathrm{H}^{\prime}\right)$,

o referido Indice ajustado nāo số relativamente à amplitude de variação como também em relação ao seu limite inferior $\left(H^{\prime}\right)$ e o número de equivalentes. 


\section{GRÁFICO 2}

CONTRIBUIÇÃO dOS DEPÓSTTOS A VISTA PARA CONCENTRAÇÃO NOS DEPÓSITOS TOTAIS

- BANCOS COMERCIAIS PRIVADOS - 1970/86

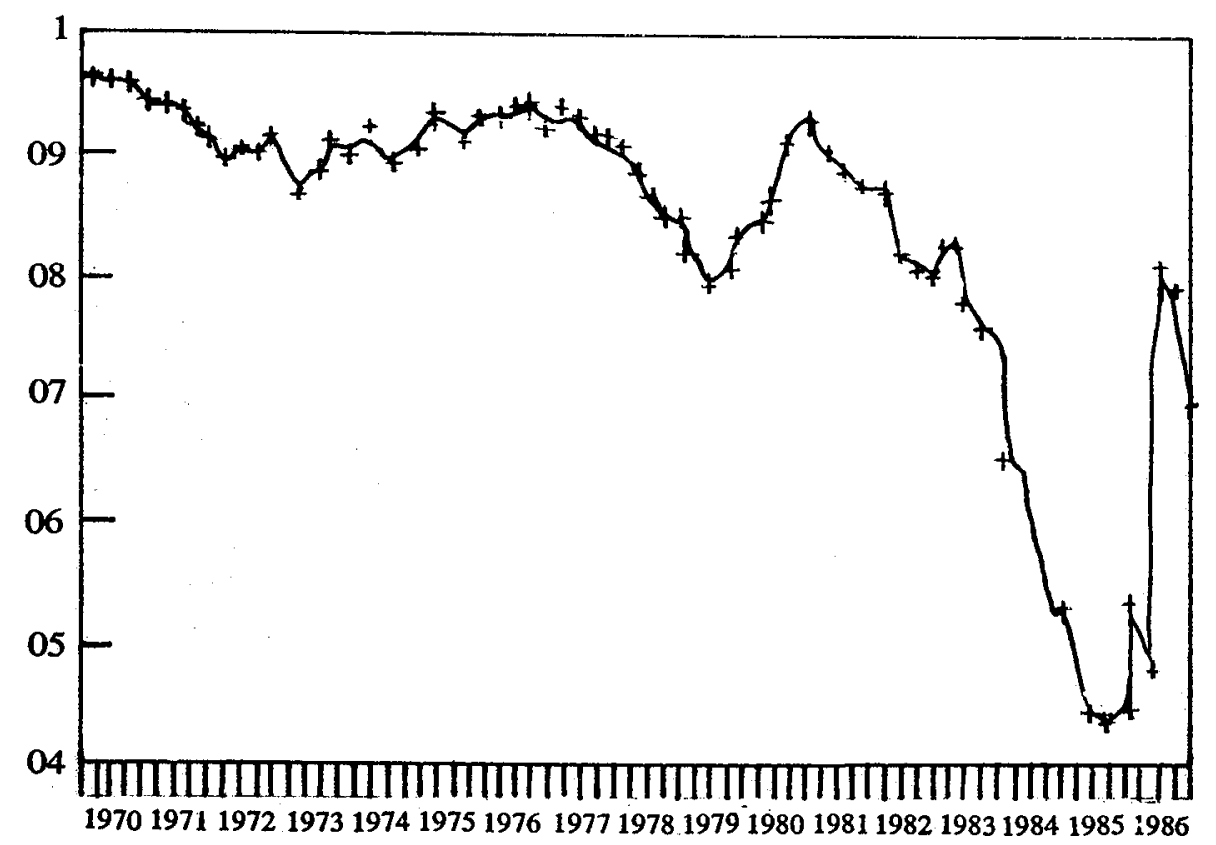

Perfodo

Cabe agora considerar isoladamente cada um os componentes dos depositos totais. E na variável depósitos a vista que se observa o maior aumento da concentração. De acordo com o gráfico 3 , constata-se um contínuo e intenso aumento do nivel de concentração, que chega a mais do que quintuplicar entre 1970 e 1986. 


\section{INDICES DE HERFINDAHL PARA DEPÓSITOS A VISTA - BANCOS COMERCIAIS PRIVADOS - 1970/86}

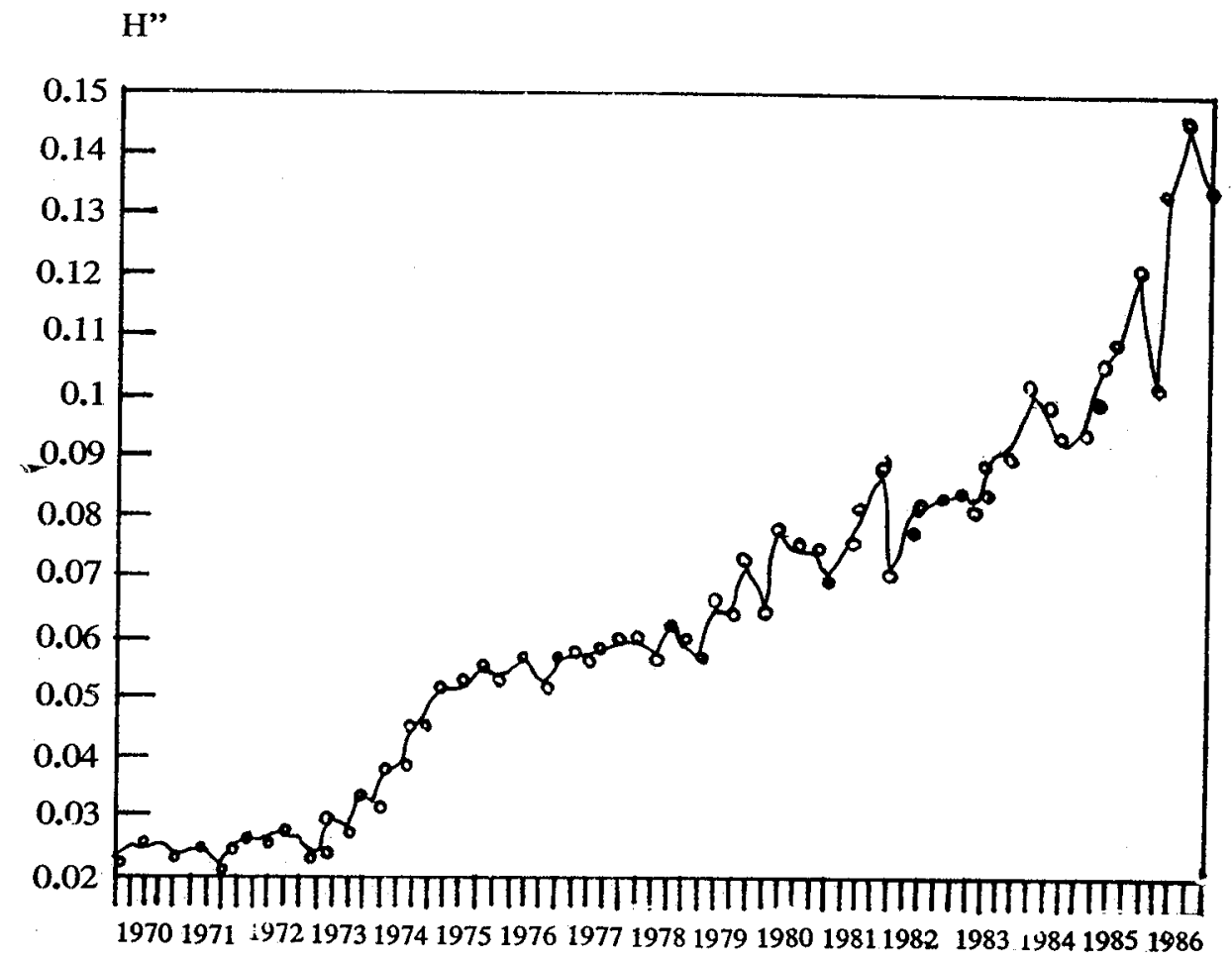

Período

Os depósitos a prazo, por outro lado, mostram um comportamento errático. É certo que entre 1970 e 1976, observa-se um grande aumento no nfvel de concentração (mais do que duplica), contudo, posteriormente, esse nfvel flutuaria seguidamente conforme se observa do grafico 4. 


\section{GRÁFICO 4}

\section{ÍNDICES DE HERFINDAHL PARA NÚMERO DE AGÊNCIAS - BANCOS COMERCIAIS PRIVADOS - 1980/87}

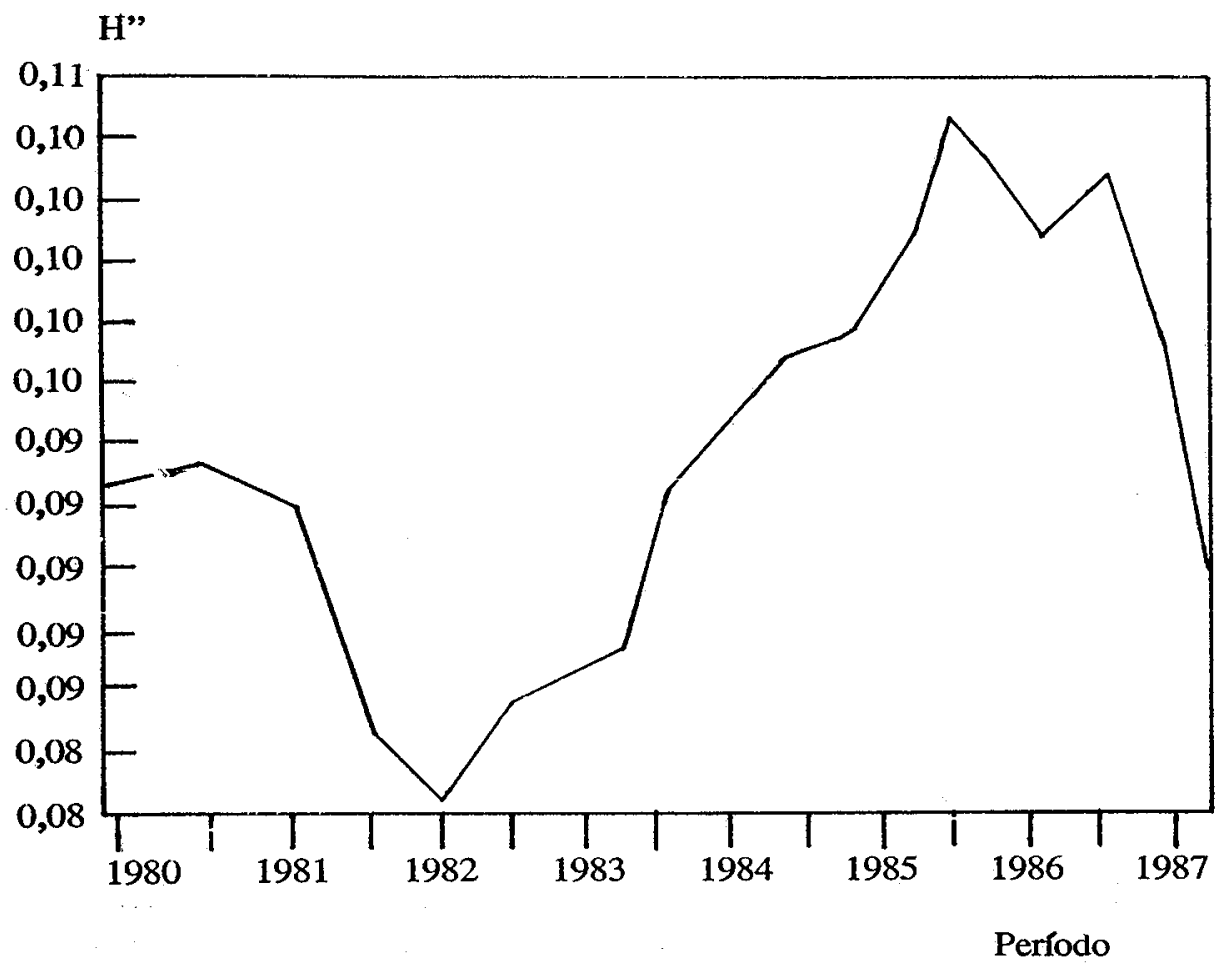

Podemos, pois, estabelecer alguns elementos de comparação entre a evolução dos dois tipos de depósitos. Os depósitos a vista apresentam uma trajetória basicamente crescente e niveis de concentração relativamente mais elevados que os depositos a prazo. A volatilidade e menor nível relativo da concentração nos depósitos a prazo não surpreende, podendo advir de um comportamento diversificador instável dos demandantes (essencialmente firmas). O nfvel sempre crescente e relativamente elevado da concentração nos depósitos a vista, é prima facie justificável pela exacerbarção de assimetrias entre bancos de tamanhos diferentes ao longo do tempo. De fato, um ambiente inflacionário no qual existem restriçōes de pagamento de juros aos depósitos a vista, induz os bancos a uma concorrência mais agressiva por esses depositos e os bancos relati- 
TABELA 2

ÍNDICES DE HERFINDAHL PARA NÚMERO DE AGÊNCIAS BANCOS COMERCIAIS PRIVADOS

\begin{tabular}{lcccc}
\hline PERÍODO & $\mathrm{H}$ & $\mathrm{H}^{\prime}$ & H' & ne \\
\hline $1980-1$ & 0,1033 & 0,1047 & 0,0910 & 9,7 \\
$1980-2$ & 0,1033 & 0,1046 & 0,0915 & 9,7 \\
$1981-1$ & 0,1026 & 0,1039 & 0,0908 & 9,7 \\
$1981-2$ & 0,0956 & 0,0968 & 0,0842 & 10,5 \\
$1982-1$ & 0,0939 & 0,0951 & 0,0822 & 10,6 \\
$1982-2$ & 0,0970 & 0,0983 & 0,0856 & 10,3 \\
$1983-1$ & 0,0986 & 0,0998 & 0,0870 & 10,1 \\
$1983-2$ & 0,1040 & 0,1054 & 0,0924 & 9,6 \\
$1984-1$ & 0,1065 & 0,1079 & 0,0946 & 9,4 \\
$1984-2$ & 0,1093 & 0,1108 & 0,0969 & 9,1 \\
$1985-1$ & 0,1104 & 0,1119 & 0,0979 & 9,0 \\
$1985-2$ & 0,1169 & 0,1186 & 0,1044 & 8,6 \\
$1986-1$ & 0,1133 & 0,1149 & 0,1009 & 8,8 \\
$1986-2$ & 0,1156 & 0,1173 & 0,1030 & 8,6 \\
$1987-1$ & 0,1106 & 0,1122 & 0,0979 & 9,0 \\
$1987-2$ & 0,1027 & 0,1042 & 0,0901 & 9,7 \\
\hline
\end{tabular}




\section{GRÁFICO 5}

INDICES DE HERFINDAHL PARA DEPÓSITOS

A PRAZO - BANCOS COMERCIAIS PRIVADOS

\section{0/86}

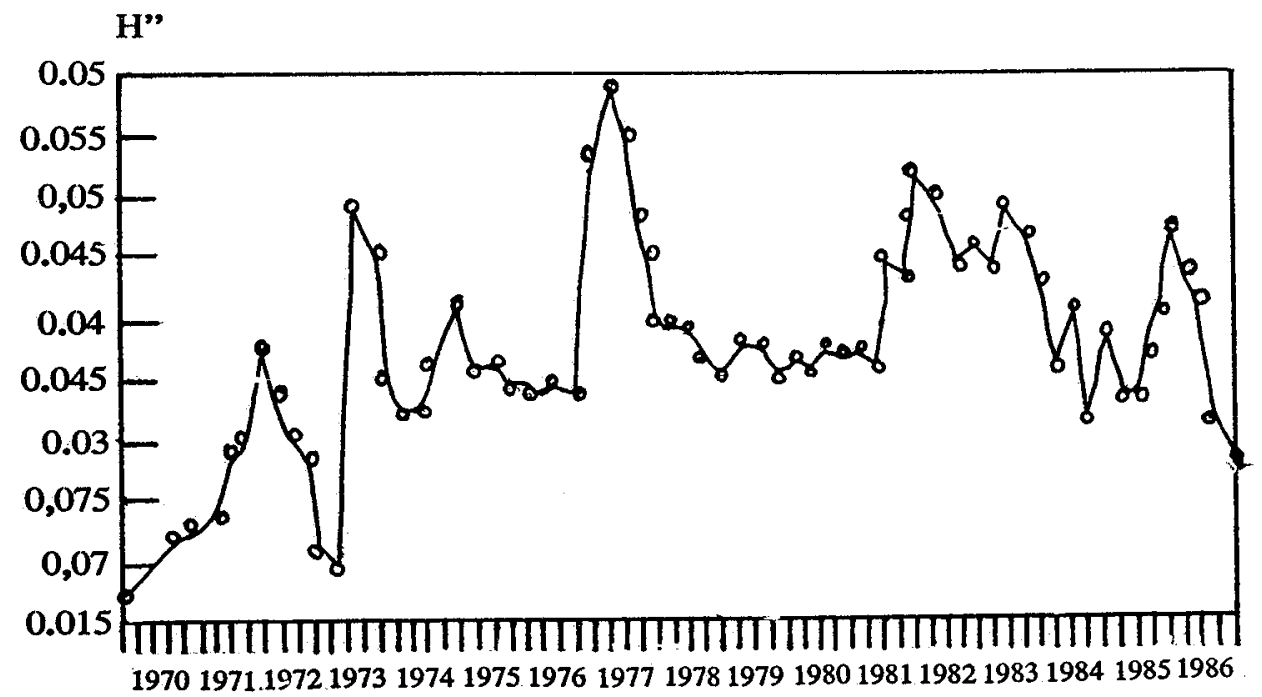

Perfodo 
vamente maiores possuem vantagem por terem uma maior rede de agências já instaladas e uma maior capacidade de expansão dessa rede. Assim, o grande incremento observadio na concentração dos depósitos a vista podem refletir fatores de cunho locacional. Nesse sentido, a tabela 2 e o gráfico 5 mostram a concentração referente ao número de agências. Pode-se constatar um ligeiro aumento na concentração, porem sర existem dados disponfveis para o perfodo 1980/87 o que não permite que concluamos inequivocamente pela relevância de assimetrias na capacidade de captação. Essas seriam corroboradas case tivéssemos uma série de fndices de concentração desde 1970 que exibisse uma tendência crescente. Outro ponto correlato, inibido pela disponibilidade de dados, refere-se a avaliar em que medida a maior capacidade de captação dos bancos maiores aumentou em função de um maior número de agências ou por conta do aumento do tamanho médio das agências. Tal ponto reveste-se de interesse, pois a ampla discussão acerca da conveniência da concentração foi referenciada à existência de economias de escala, e essas em parte relacionam-se ao tamanho médio das agências (Ver Alves/1974).

Por fim, cumpre estudarmos a trajetória da concentração nos empréstimos. Para tanto, lancemos mão da tabela 3 e do gráfico 6, que permitem constatar um amplo aumento da concentração que mais do que dobra. Outrossim, há que se destacar que, semelhantemente as variáveis de depósitos, pode-se observar um ritmo de elevação da concentração particularmente elevado até meados da década de setenta, perfodo este caracterizado por um intenso incentivo a concentração por parte da $\mathrm{CO}$ FIE. Adicionalmente, vale mencionar que o nfvel de concentração desta variável revela-se sistematicamente mais baixo que o referente aos depósitos a vista, o que pode decorrer de um comportamento diversificador dos grandes bancos, que inseridos em conglomerados, não centralizariam suas aplicações em emprésuimos por possufrem um maior espectro de alternativas de aplicações. Os bancos de pequeno porte teriam, ao contrário, uma maior dependência dos emprestimos enquanto aplicação. ${ }^{6}$

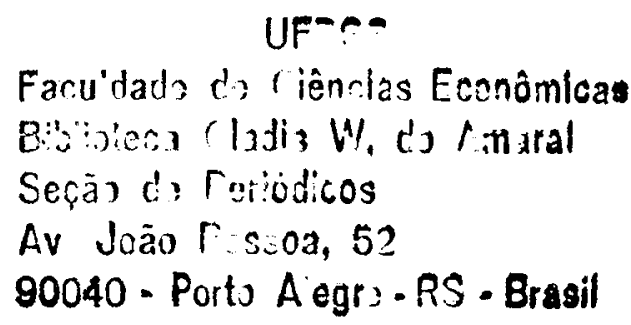

6 Vale lembrar que Tavares (1983) aponta para um aumento da importancia da carteira de câmbio nas aplicaçóes dos grandes bancos entre 1975 e 1981. 


\section{GRÁFICO 6}

INDICES DE HERFINDAHL PARA EMPRESTIMOS

- BANCOS COMERCIAIS PRIVADOS - 1970/86

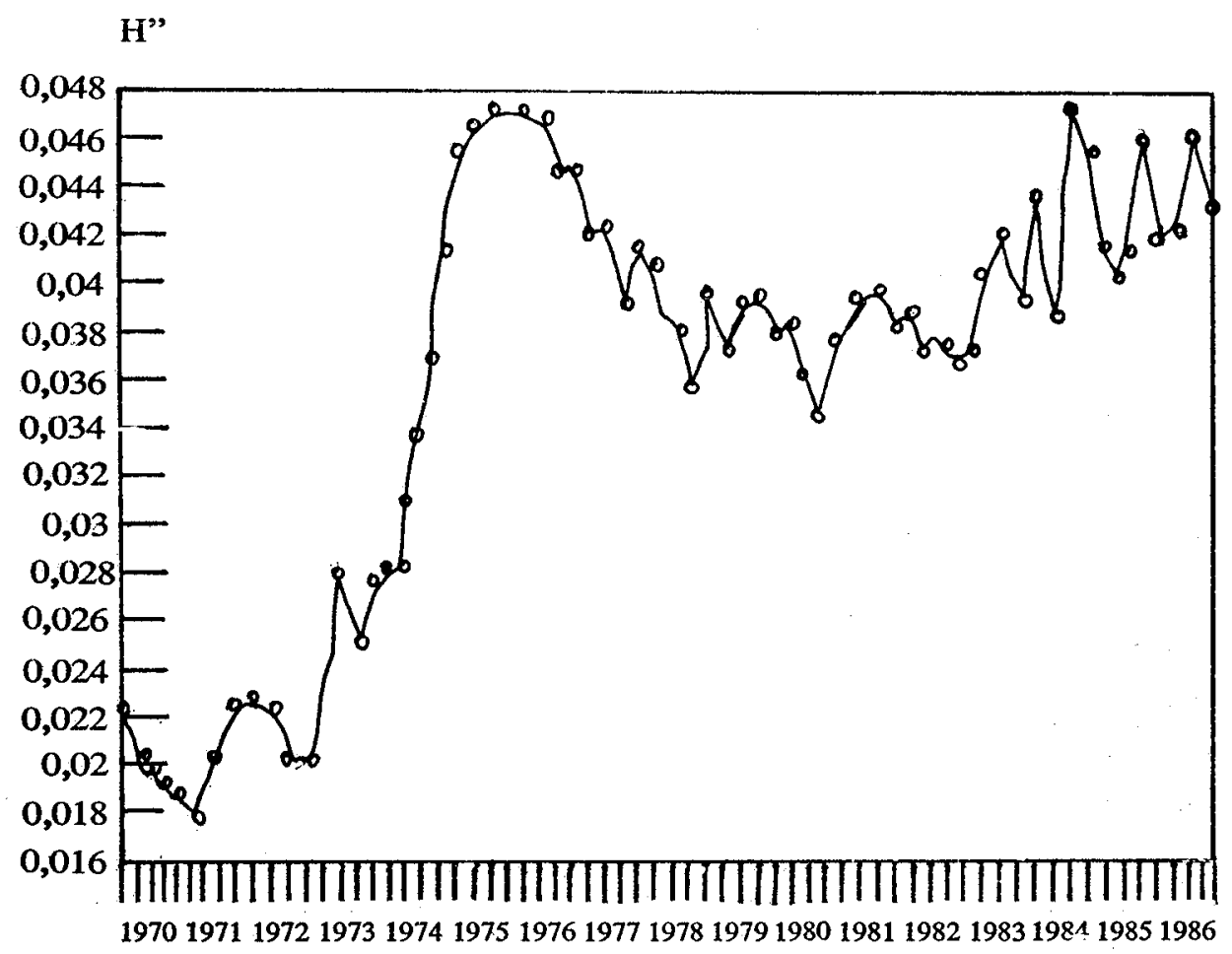




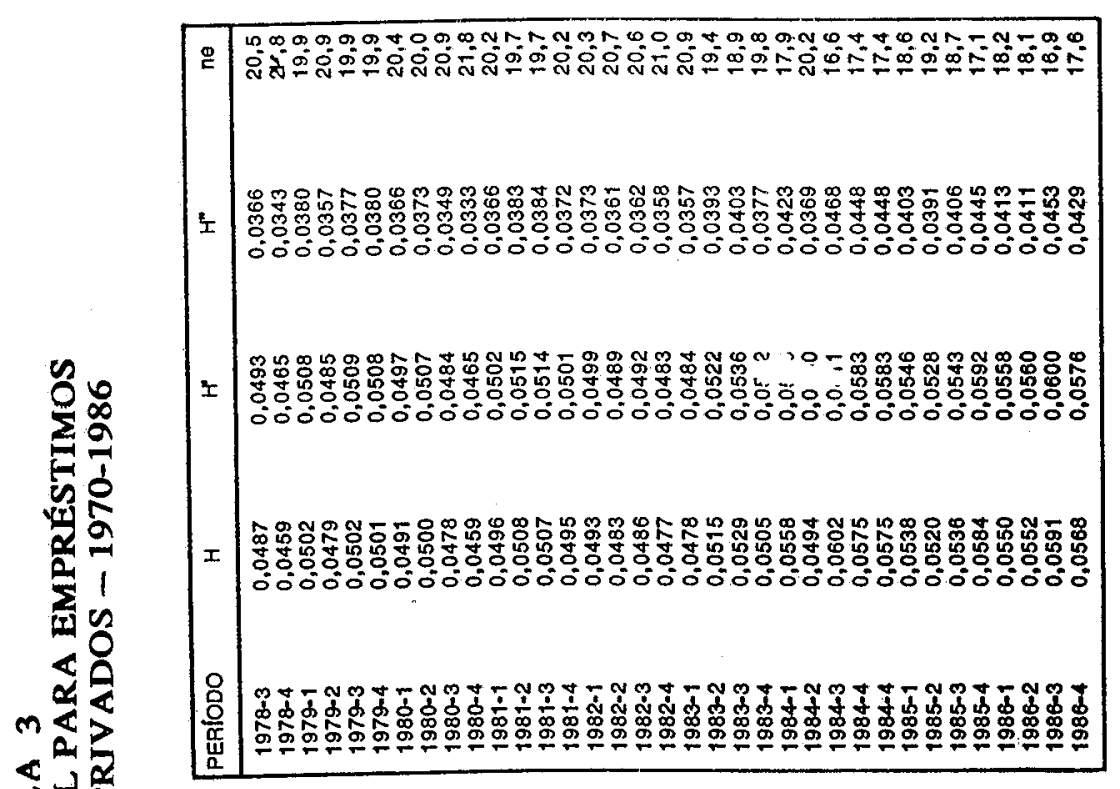

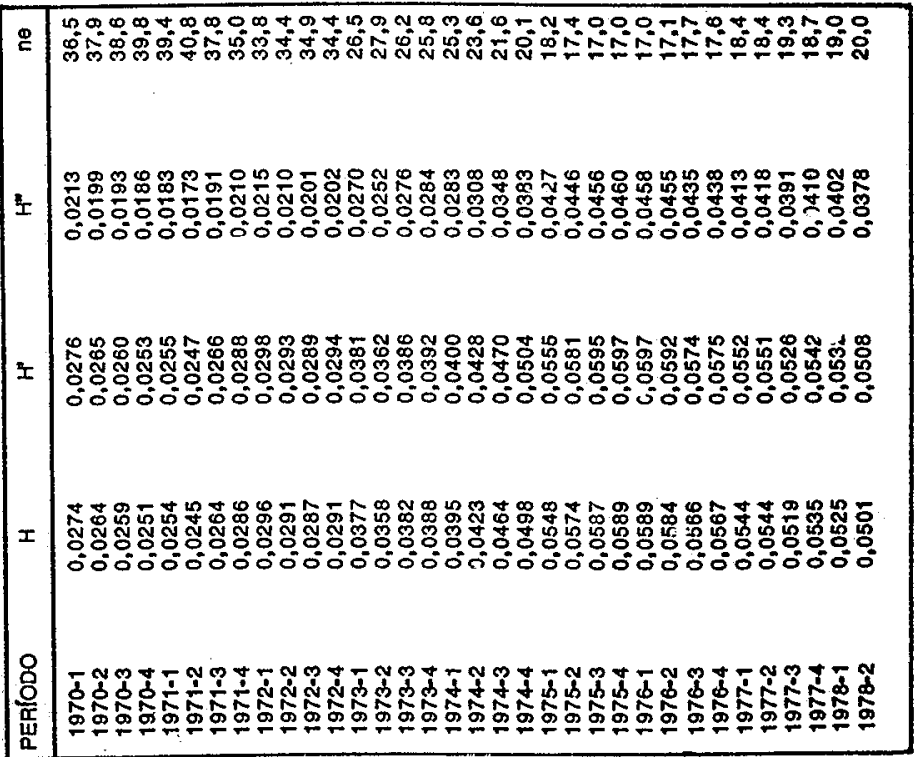




\section{CONSIDERAĢŌES FINAIS}

$O$ presente trabalho procurou fornecer um mapeamento descritivo da estrutura de mercado do setor bancário brasileiro, aproximada em termos da evolução da concentração. Nesse sentido, empreendeu-se um esforço cuidadoso de quantificação da mesma. A consideração do segmento de bancos privados permitiu concluir por um sensivel aumento no nfvel de concentração. Com efeito, além das medidas governamentais de incentivo à concentração, o próprio ambiente inflacionário tende a induzir os bancos a novas formas de concorrência, segundo dois movimentos:

i) aumento da competição por depósitos a vista - nesse caso, a forma típica de competição foi a de oferecer vantagens não pecuniárias, dentre as quais se destaca a localização especial das agências;

ii) oferecimento de ativos financeiros alternativos aos depósitos a vista - aqui estamos nos referindo indiretamente ao processo de corglomeração que se acentuaria em meados da década de setenta (Ver Perdigão/1983).

No processo de conglomeração, os aspectos locacionais desempenham um papel importante, na medida em que as agências dos bancos comerciais funcionam como postos de captação integrada dos conglomerados financeiros. Assim, face a essas mudanças nas formas de concorrência no setor bancário, espera-se que bancos de menor porte explorem mais intensamente as oportunidades que não sejam muito demandantes quanto à extensão da rede de agências. Tal certamente $\boldsymbol{\epsilon}$ o caso dos depósitos a prazo, nos quais constatou-se com efeito um nfvel de concentação relativamente mais baixo, o que pode ser explicado face ao perfil dos demandantes (essencialmente firmas) para os quais o fator locacionai não e fundamental como nos depósitos a vista. De fato, pode-se dizer que os bancos atuam como atacadistas nos depósitos a prazo.

Isso posto, cumpre tecer considerações, de cunho prospectivo sobre as conseqüêricias da redução da taxa de inflação sobre a configuração do setor bancário. Nesse sentido, conforme se observou após o Plano Cruzado, a repentina redução do nfvel da inflação levou a ajustes por parte dos grandes bancos em termos do fechamento de algumas agências e, atualmente, é corriqueira a cobrança de taxas de serviços bancários como extratos e talóes de cheque. Pode-se prever que, caso a economia brasileira entre numa trajetória de estabilidade de preços, haja uma leve redução no nível de concentração nas variáveis em que o fator locacional é relevante (depósitos a vista e número de agências por exemplo) dada a redução do número de agências dos bancos de maior porte e/ou uma intensificação da cobrança de taxas de serviço. Pode-se esperar, portanto, que o eventual controle da inflação contribua (ainda que pouco) para 
o aumento no grau de concorrência no setor.

Outro ponto de interesse refere-se a maior facilidade de entrada representada pelo advento dos bancos múltiplos. Nesse caso, pode-se esperar alguma redução da concentração naquelas variáveis nas quais o aspecto locacional não seja fundamental, já que vários dirigentes desses novos bancos revelaram não pretenderem competir no varejo.

\section{BIBLIGRAFIA}

ALVES, J.B. Fatores Determinnntes da Eficiencia dos Bancos Comerciais. Rio de Janeiro: Sindicato dos Bancos do Estado da Guanabara, 1974.

BARBOSA, F.H. Medidas de Concentração. Revista de Econometria, v.1, n.1, 1981.

BOUZAN, A. Bancos Comrciais no Brasil: Uma Anflise do Desenvolvimento Recente - 1967/71, São Paulo: Federação Brasileira de Associaçóes de Bancos, 1972.

CARVALHeIRO, N. Bancos Comerciais no Brasil - 19641976: Creacimento e Concentraçó, São Paulo: IPEUUSP, 1982. (Dissert., mestr. econ.).

CASTRO, N.O.P. As Causa Econbmicas da Concentragro Bancfíria, Rio de Janeino: IBMEC, 1981.

CORRÊA, V.P. Concentraçio e Lucratividade dos Bancos, Braşía: Universidade de Brasflia, 1985. (Dissert., mestr. econ.).

FONSECA, J. \& A.Z. SANVICENTE, A.Z. A Concentração do Sistema Bancário Brasileiro. Revista Brasileira de Mercado de Capitaix, v.3, n.9, 1977.

MARQUES, N.S.F. A Concentraçăo do Capital Bancário no Brasil (1964-1984), Reviata Brasileira de Mercado de Capitais, v.11, n.3, 1985.

MEIRELLFS, A.C. A Evoluçío da Estrutura do Sistema Bancfrio Bravileiro no Perfodo 1966-74. Rio de Janeiro: Sindicato dos Bancos do Estado da Guanabara, 1975.

MELO FIL HO, L.C. Ao Longo do Tempo a concentraçẵo. Jornal do Brasil, mar. 1972.

PERDIGÁO, L.A. Conglomerac̃os Financeiros: Anflise e Desempenho no Brasil no Perfodo 1978/81. Rio de Janeiro: IBMEC, 1983.

RESENDE, M. Estruture de Mercado no Setor Bancirio: Determinuntes e Implicaf̧óes sobre a Polf́tica Monetíria. Rio de Janeiro: PUC/RJ, 1989. (Dissert, mestr. econ.).

Con.). Medidas de Concentraço Industrial: Uma Introdaçăo. Rio de Janeiro: FEA/ÚFRJ, 1990. (Texto Didático n⿳0 44).

SRIVASTAVA, D. \& AGGARWAL, P.K. Messuring Tax Revenue Centralization in Federal Fiscal Systems: a Case Study of Inüia. Public Finance, v. 34, n.3, 1979.

TAVARES, M.A.R. Juros, Custos c Concentr=-z5o Bancíria no Brasil 1967r76, Sto Paulo: IPE $\int U S P, 1981$. (Dissert., mestr. econ):

Cre , \& CARVALHEIRO, N. O Setor Bancírio Brasileiro: Algana Aspoctos do Crescimento e da Concentraçio. São Paulo, IPE/USP, 1985.

\section{ÁBSTRACT}

This article aims at quantifying the banking concentration in Brazil along the period 1970/86 for the variables total deposits, demand deposits, time deposits, number of branches and loans. One can verify a large increasing in the concentration in the group of frivate commercial banks, what reflects incentive measures from the government in one hand, and the changing nature of the competition forms on the other hand, due to the inflationary environment. One can expect, that price stabilization would contribute, yet if not much, to increase the degree of competition in the sector. 